\title{
UDOT Signal Performance Metrics: New and Upcoming Metrics
}

\section{Jamie Mackey}

UDOT Statewide Signal Engineer jamiemackey@utah.gov 


\section{Purdue Split Failure}

\section{Purdue Split Failure}

SIG\#7219 - Foothill \& Sunnyside

Phase 5

11:00 a.m. to 1:00 p.m.

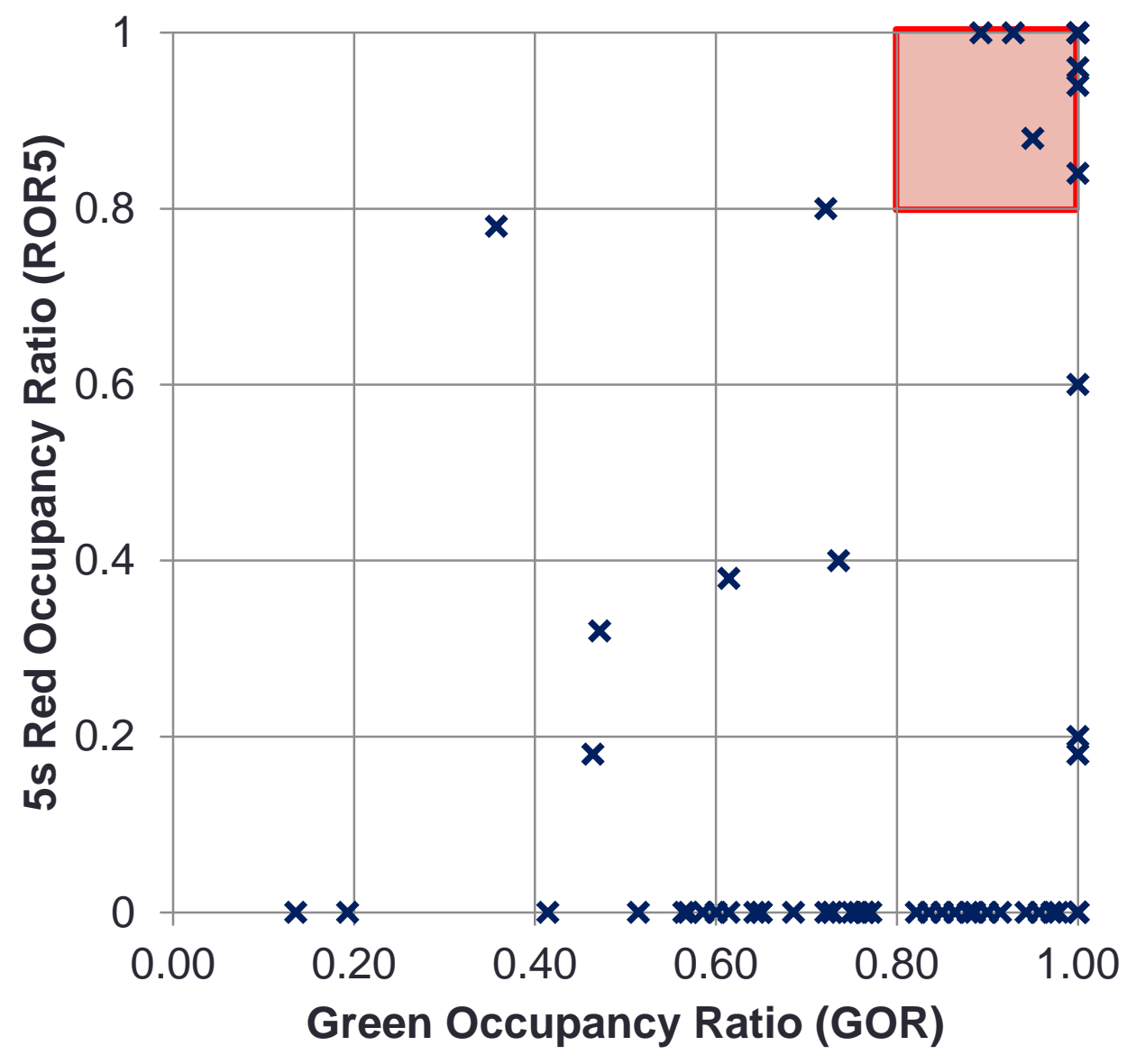

EVENT CODES

1 - Green Start

8 - Yellow Start

9 - Red Start

10 - Phase Termination

4-6 - Termination Type

81 - Detector Off

82 - Detector On

Split Fail Rate: 16\% (9/57 cycles)

Max Consecutive Failures: 3 


\section{Purdue Split Failure - 24-hr format}

Foothill Drive Sunnyside Signal 7219 Phase: 5 Southbound

Tuesday. September 01.2015 12:00 AM-Wednesday. September 02, 2015 12:00 AM

Plan 10

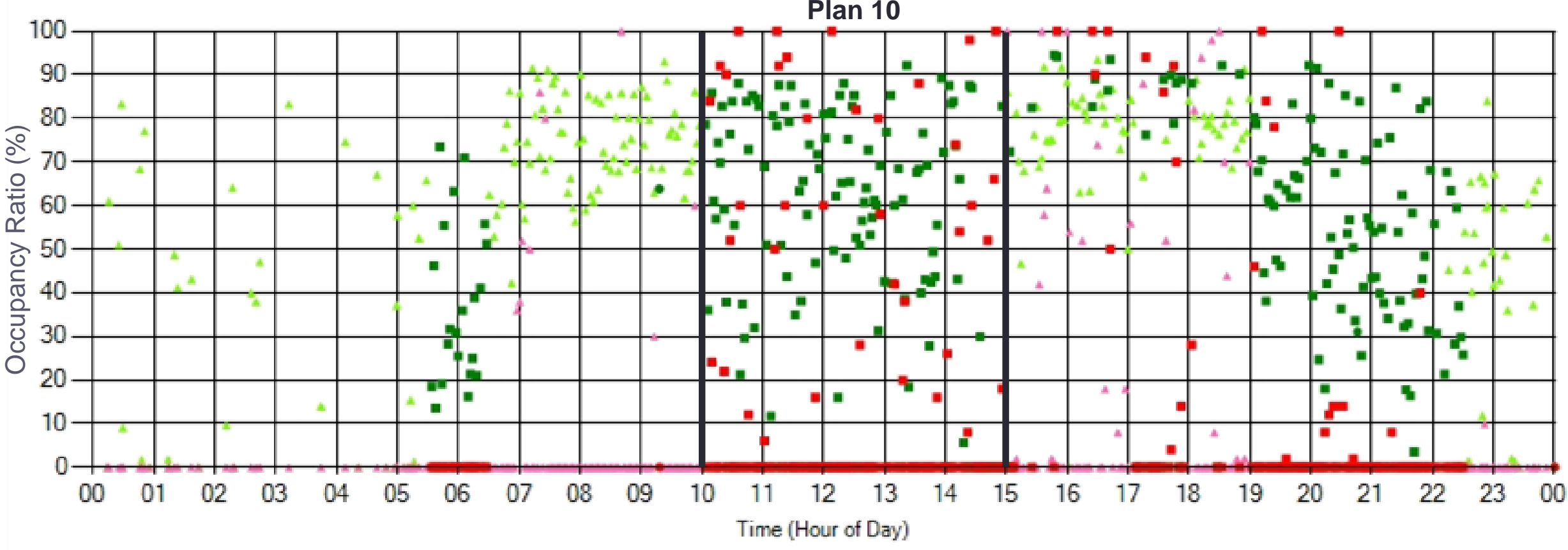

\footnotetext{
- GOR-MaxOut

GOR-GapOut

- GOR-ForceOff

- GOR-Unknown

- ROR - MaxOut

- ROR - GapOut

- ROR-ForceOff

- ROR - Unknown
} 


\section{Purdue Split Failure - 24-hr format}

Foothill Drive Sunnyside Signal 7219 Phase: 5 Southbound

Tuesday. September 01.2015 12:00 AM-Wednesday. September 02.2015 12:00 AM

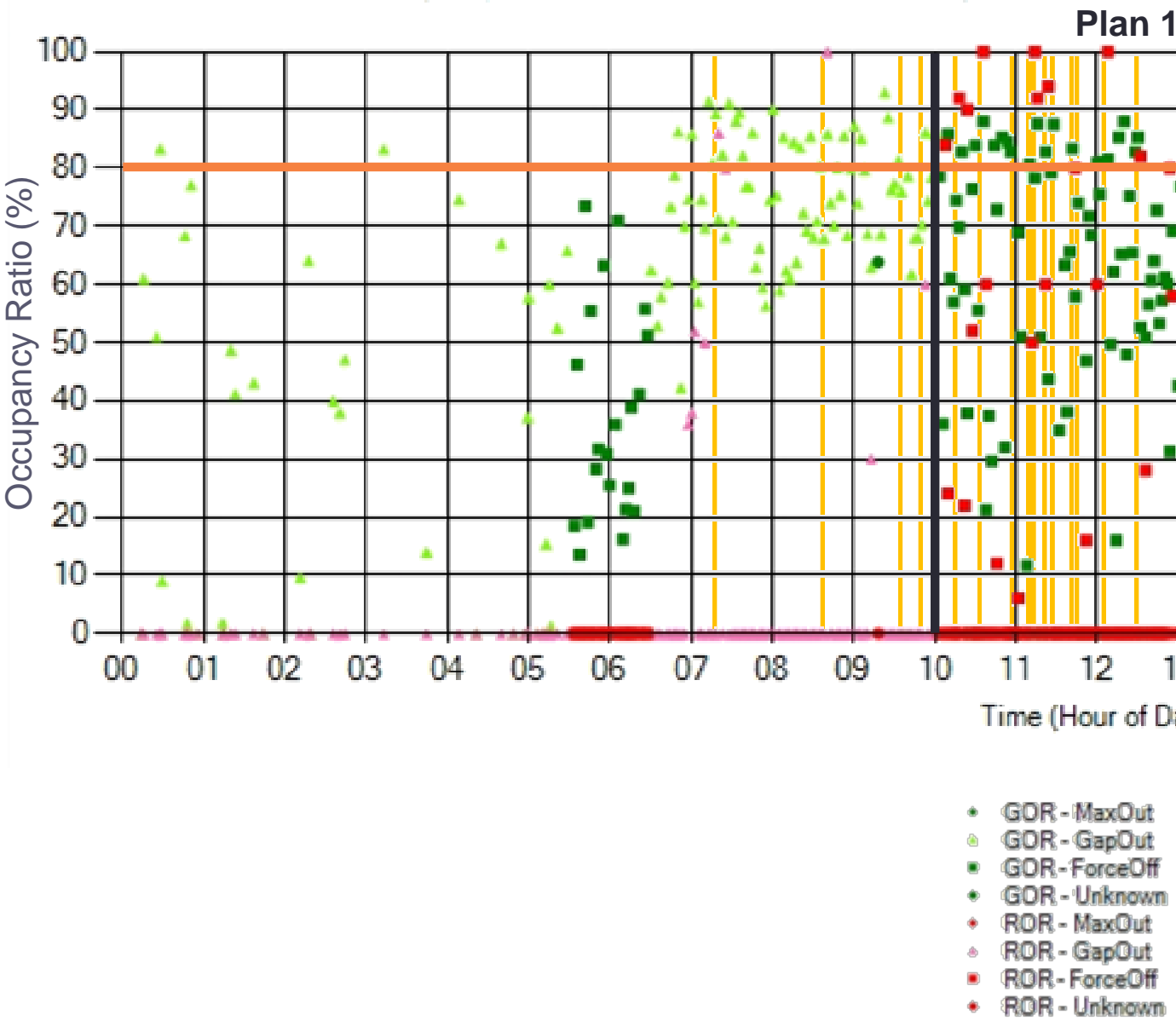




\section{Purdue Split Failure - 24-hr format}

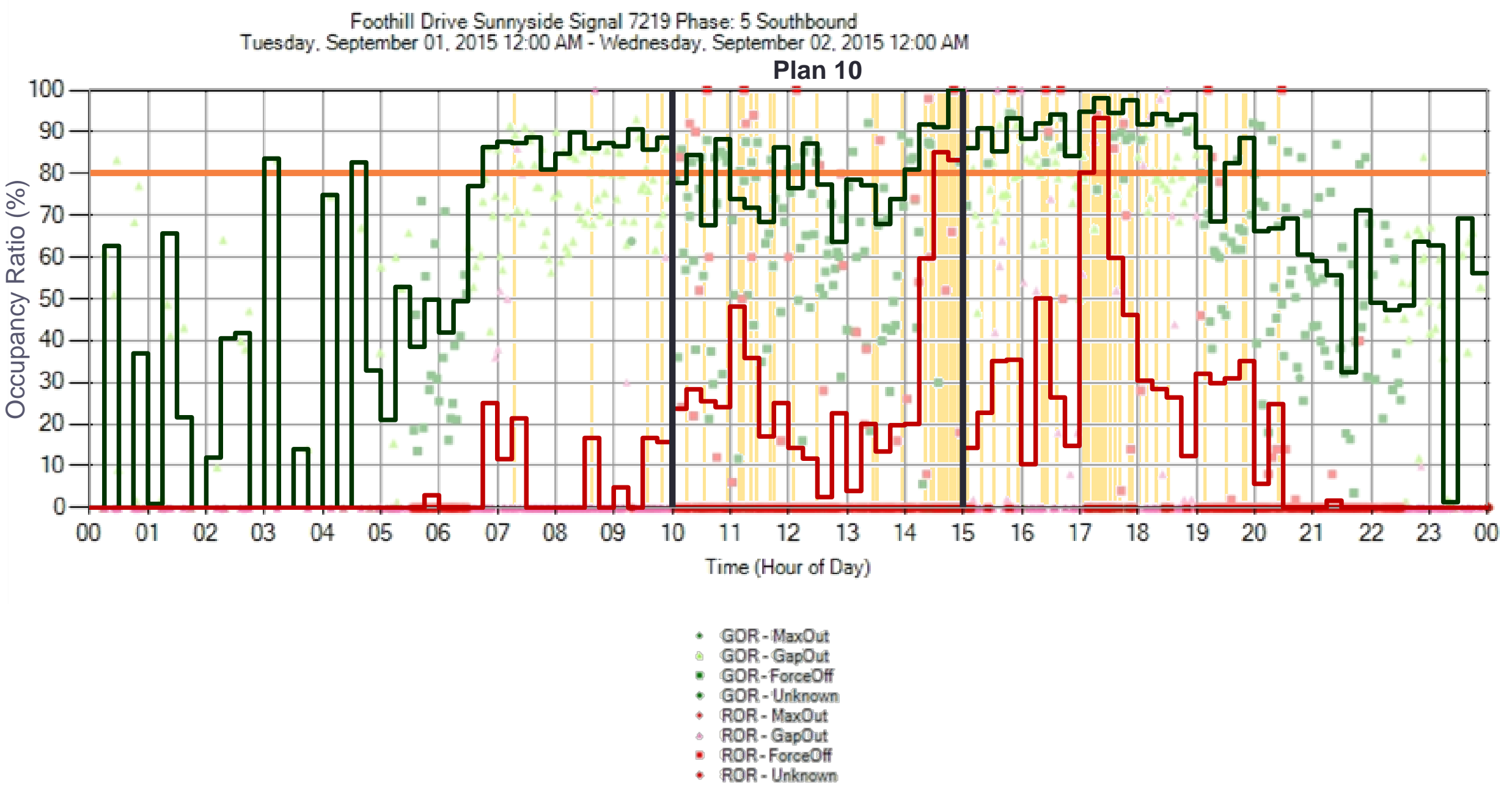




\section{Detection for Purdue Split Failure}

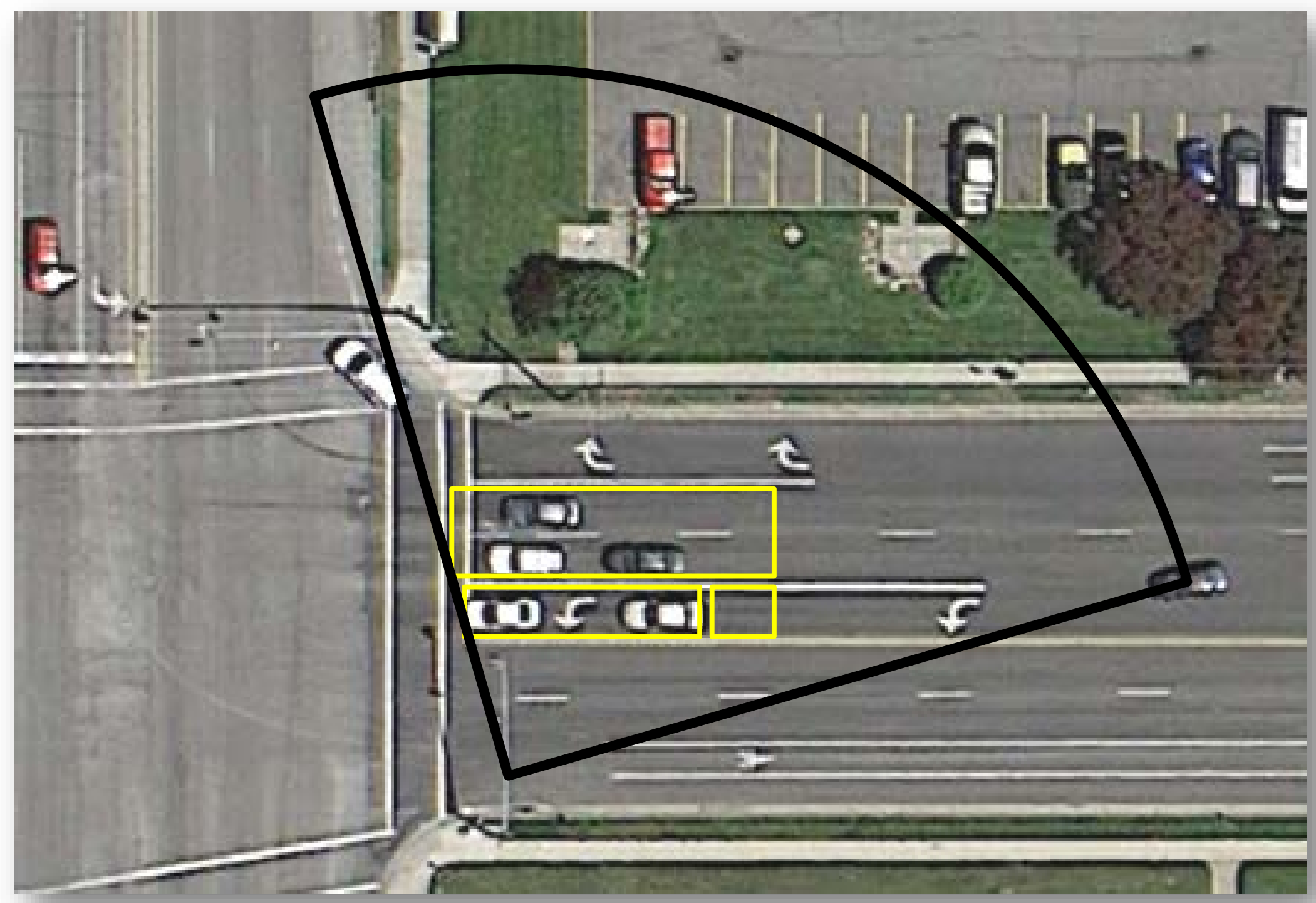




\section{New Metrics}

-Metric Type

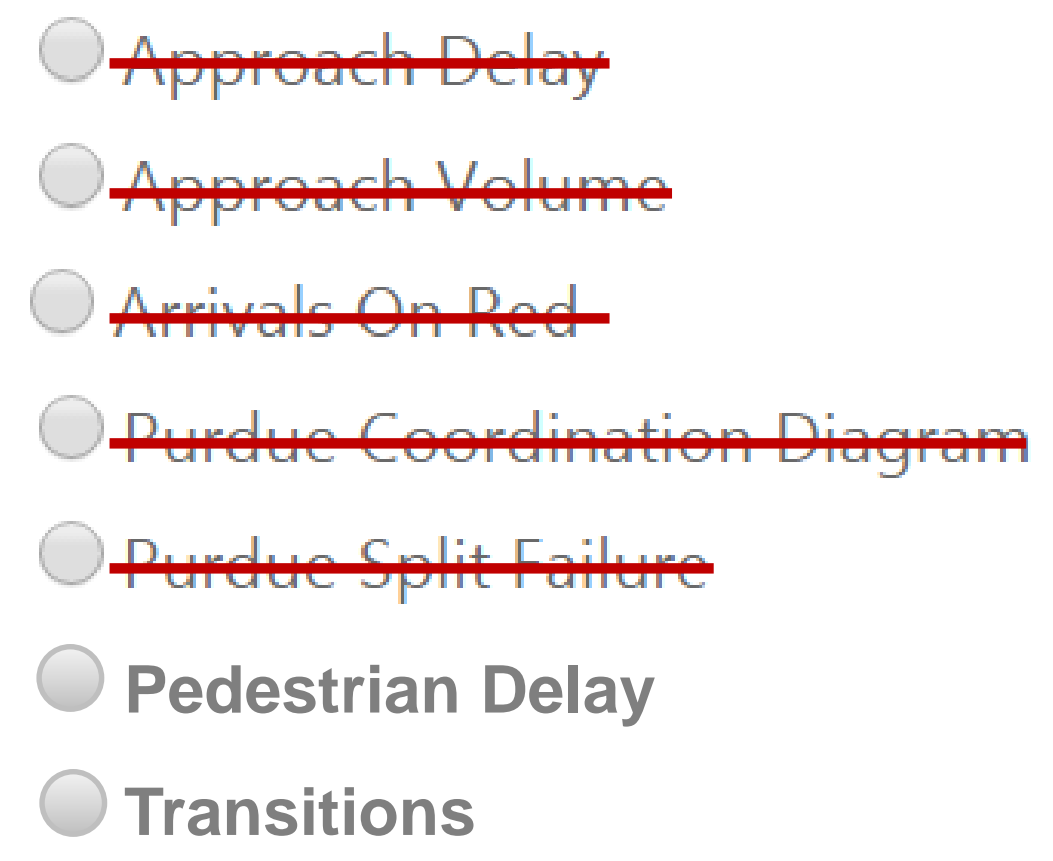

- Purdue Phase Termination

speed

Split Monitor

Fuming Novement Counts

Yellow and Red Actuations

Preemption Details

Transit Signal Priority Details 


\section{Pedestrian Delay}

EVENT CODES

45 - Ped Call on

21 - Ped Walk on

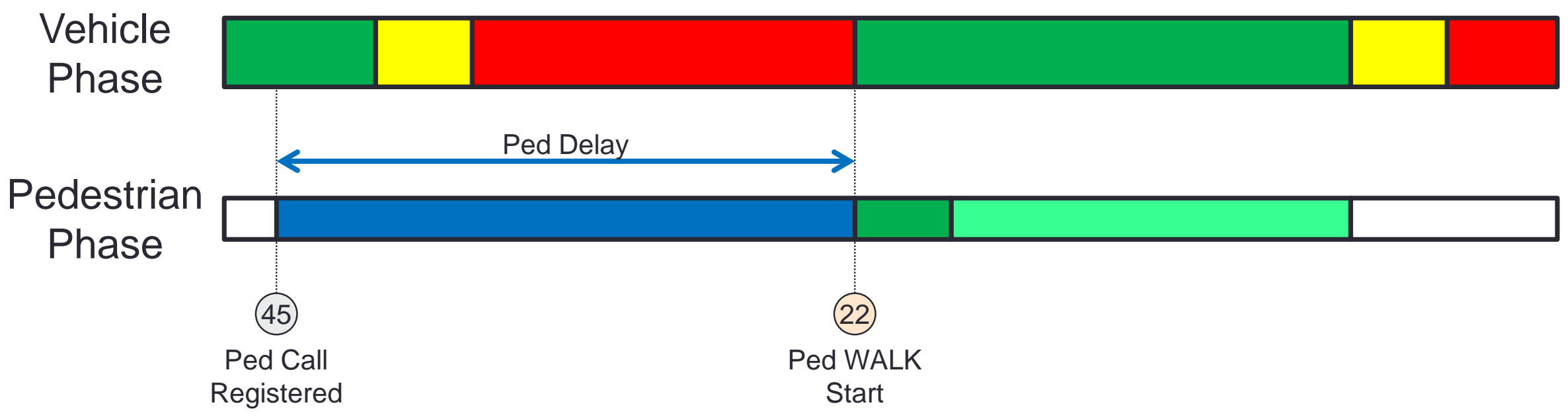




\section{Pedestrian Delay}

EVENT CODES

45 - Ped Call on

Tuesday. September 01, 2015 12:00 AM - Wednesday. September 02, 2015 12:00 AM

21 - Ped Walk on

167-Ped Acutations(PA) 00:00-Min Delay 01:20-Max Delay 00:30-Average Delay(AD)

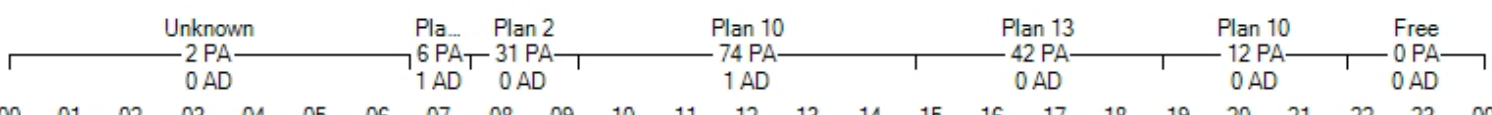

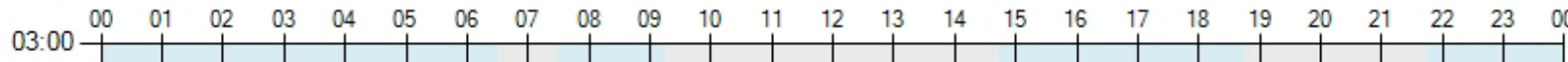

Phase 2

Coordinated phase

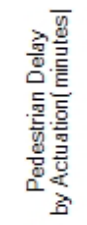

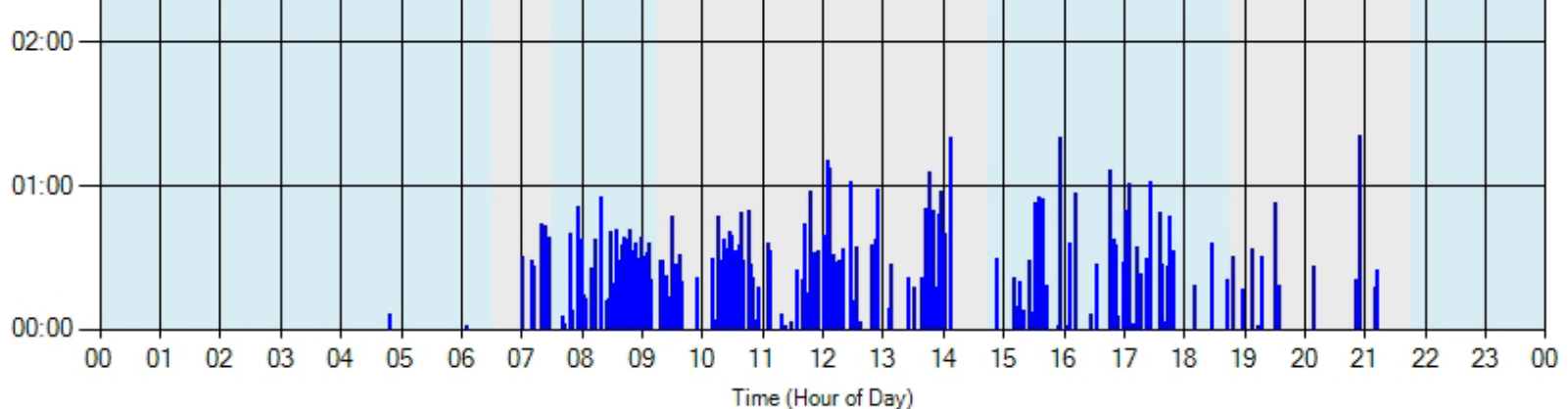

Phase 4

Side street

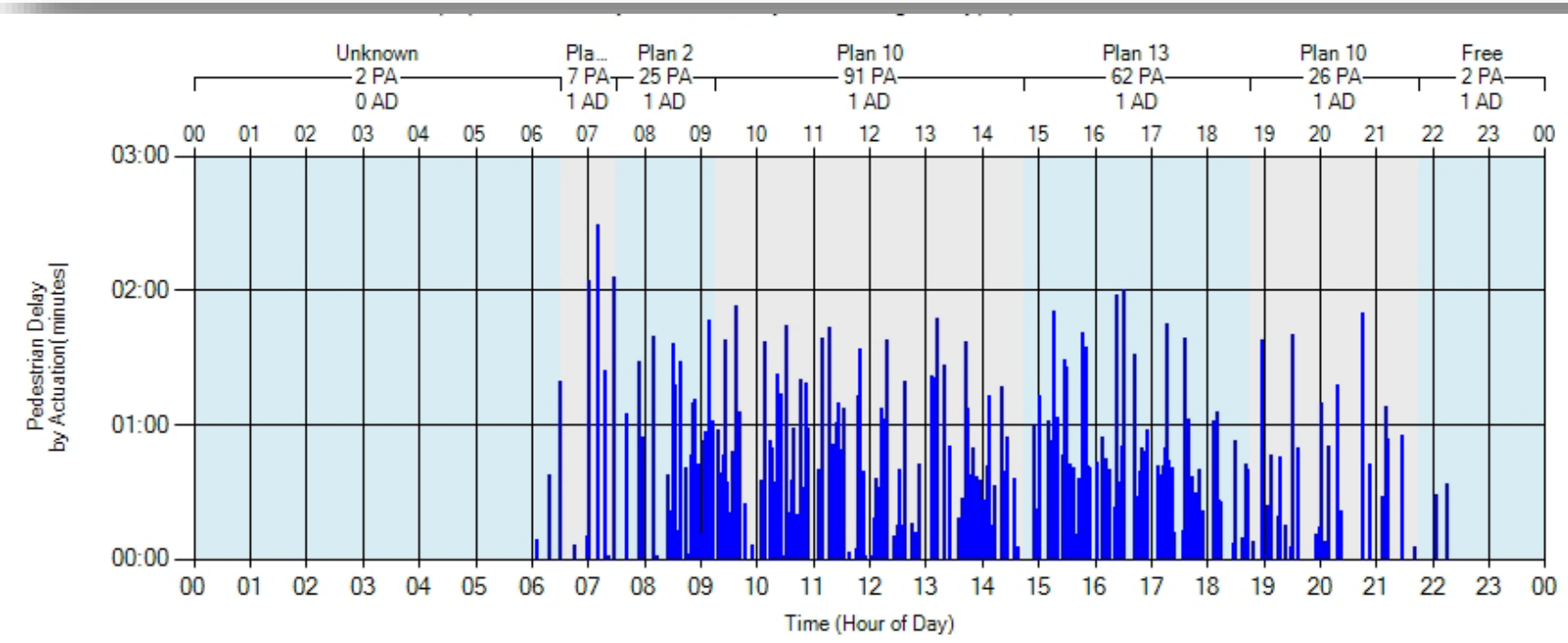




\section{Transition}

\section{EVENT CODES}

150, Param 1 - In Step

150, Param 2 - Transition Add

150, Param 3 - Transition Subtract

150, Param 4 - Transition Dwell

State St \& Orem Center St - SIG\#6311

$12 / 9 / 20150: 00$ to $12 / 9 / 2015$ 23:59

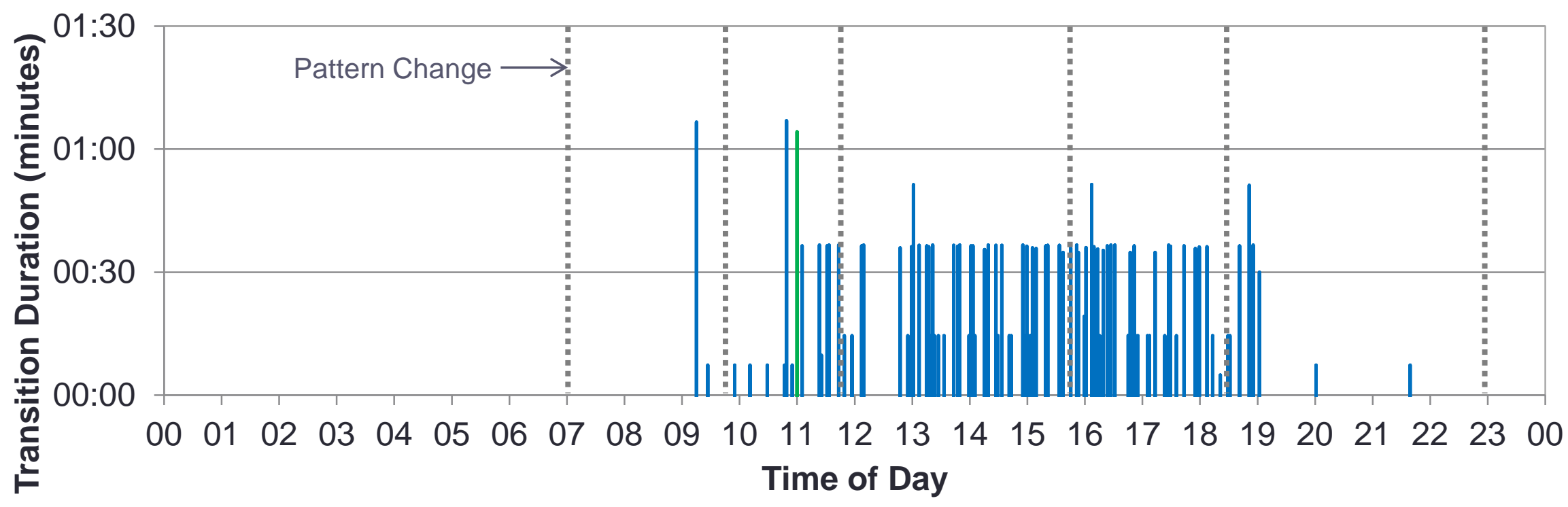

—Add — Subtract —Dwell 


\section{Transition}

\section{EVENT CODES}

150, Param 1 - In Step

150, Param 2 - Transition Add

150, Param 3 - Transition Subtract 150, Param 4 - Transition Dwell

State St \& Orem Center St - SIG\#6311

$12 / 9 / 20150: 00$ to $12 / 9 / 2015$ 23:59

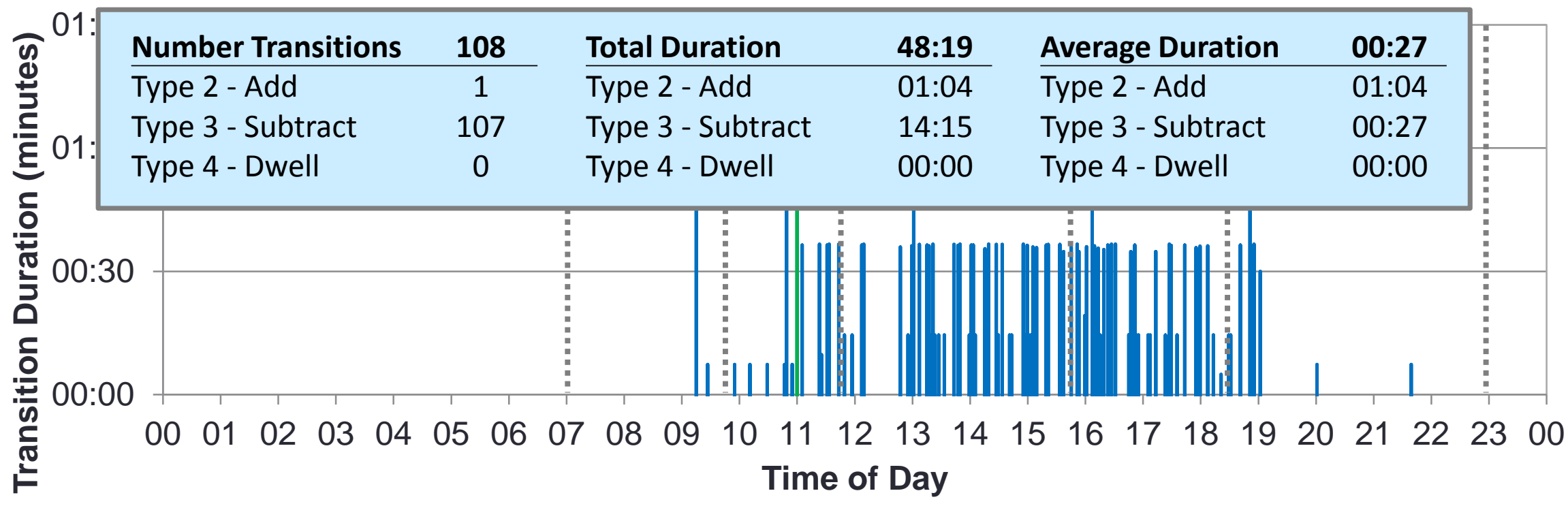

—Add — Subtract —Dwell 


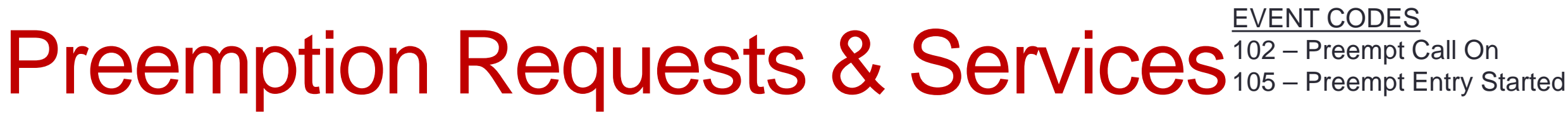

Wednesday, December 09, 2015 12:00 AM - Wednesday. December 09. 2015 11:59 PM

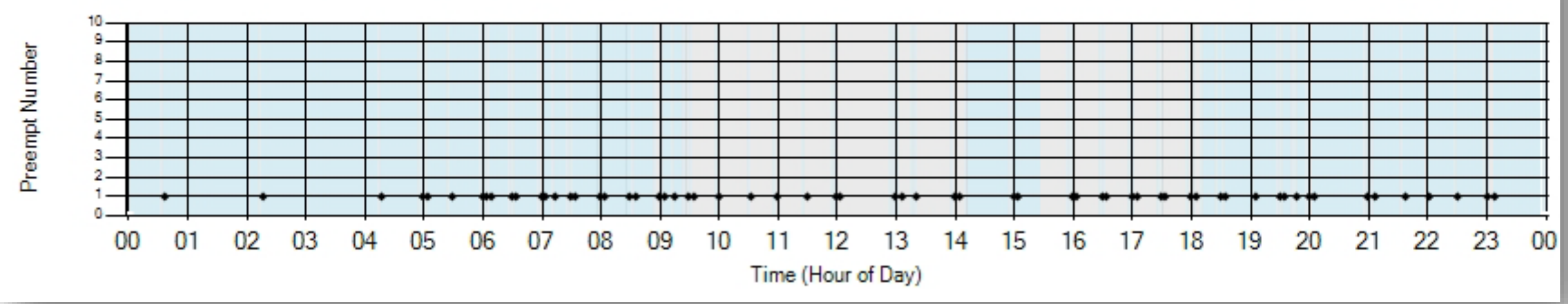

Preempt Service Chart for Geneva Road 400 South Signal 6312

Wednesday. December 09, 2015 12:00 AM - Wednesday. December 09, 2015 11:59 PM

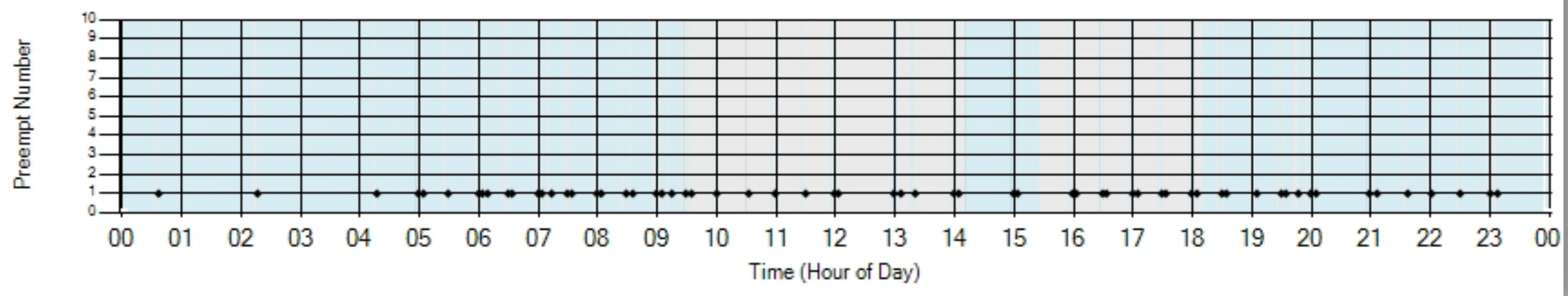




\section{Preemption Details (Rail)}

EVENT CODES

102 - Preempt Call On 103 - Gate Down 104 - Preempt Call Off 105 - Preempt Entry Started 106 - Begin Track Clearance 111 - Begin Exit Interval 150, Param 1 - In Step

\section{Preemptor \#1 Event Details}

Geneva Pkwy \& 400 S - SIG\#6312

12/9/2015 0:00 to 12/9/2015 23:59

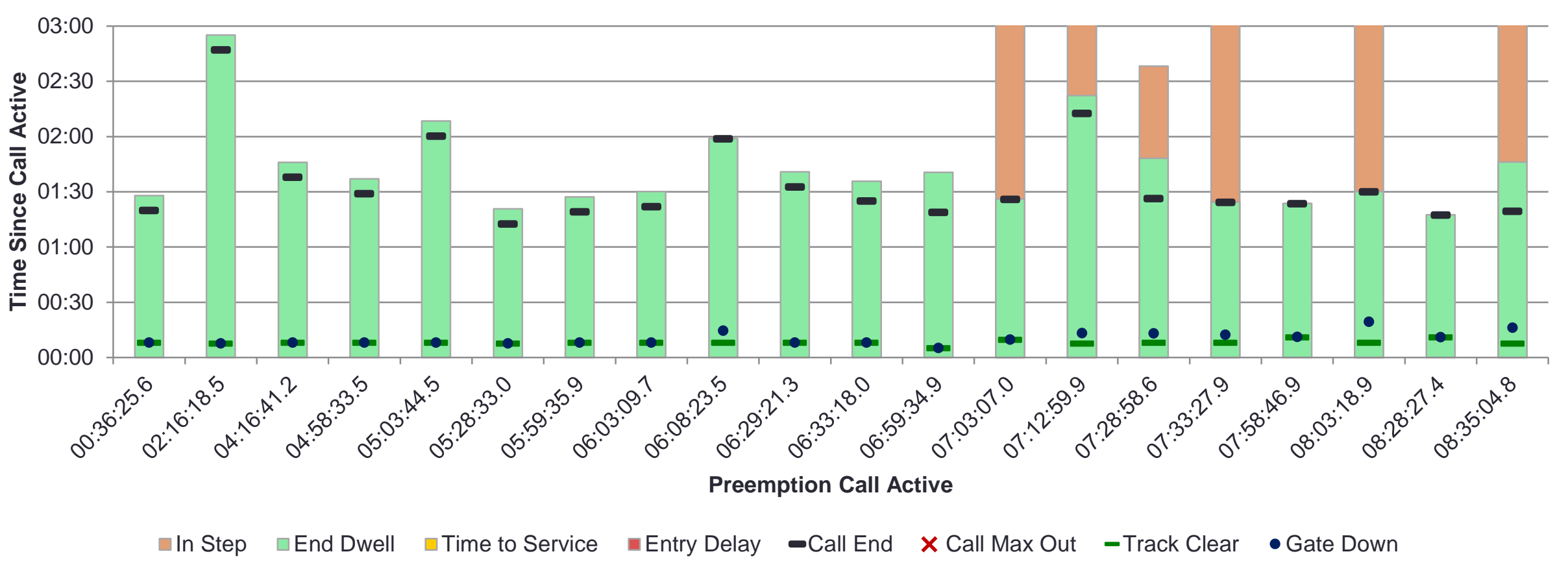




\section{Preemption Details (Rail)}

\section{EVENT CODES}

102 - Preempt Call On

103 - Gate Down

104 - Preempt Call Off

105 - Preempt Entry Started

106 - Begin Track Clearance

111 - Begin Exit Interval

150, Param 1 - In Step

\section{Preemptor \#1 Event Details}

Geneva Pkwy \& 400 S - SIG\#6312

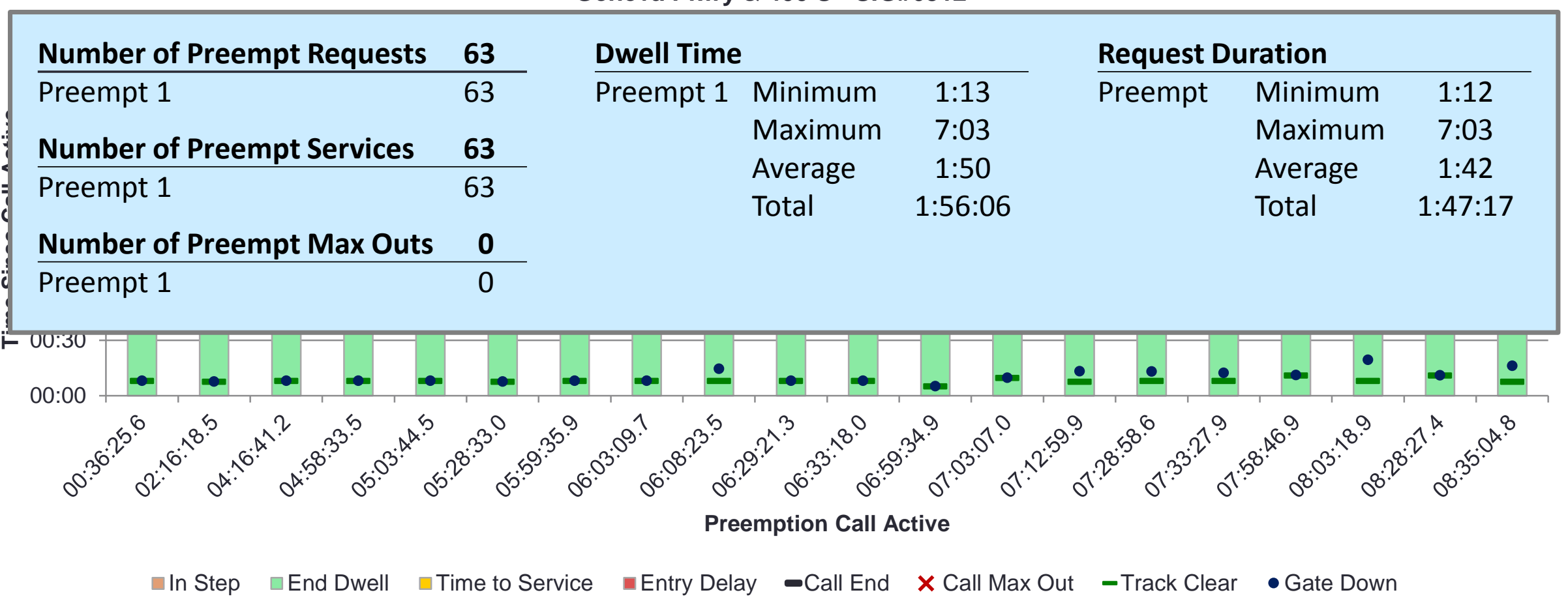




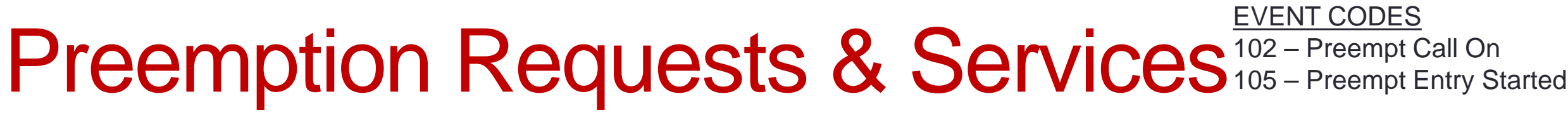
Saturday, December 05, 2015 12:00 AM - Sunday, December 06, 2015 12:00 AM

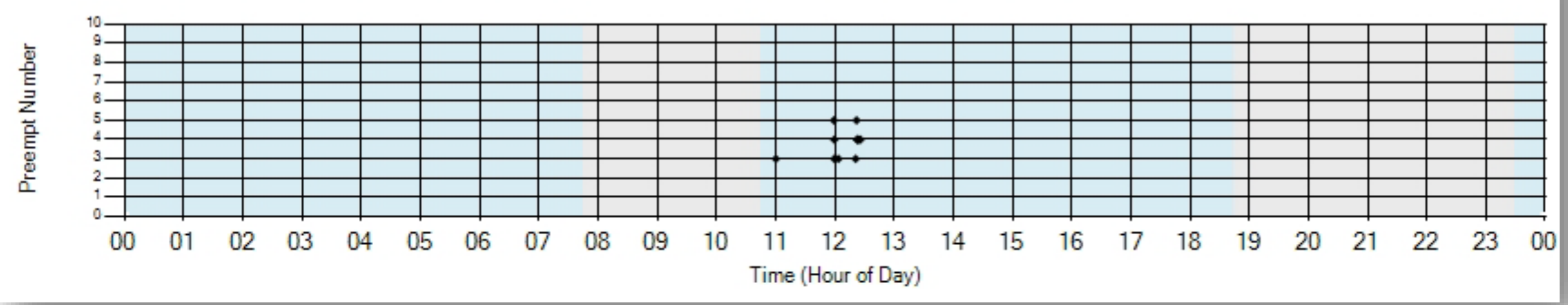

Preempt Service Chart for State Street Center Street Signal 6311

Saturday, December 05, 2015 12:00 AM - Sunday, December 06, 2015 12:00 AM

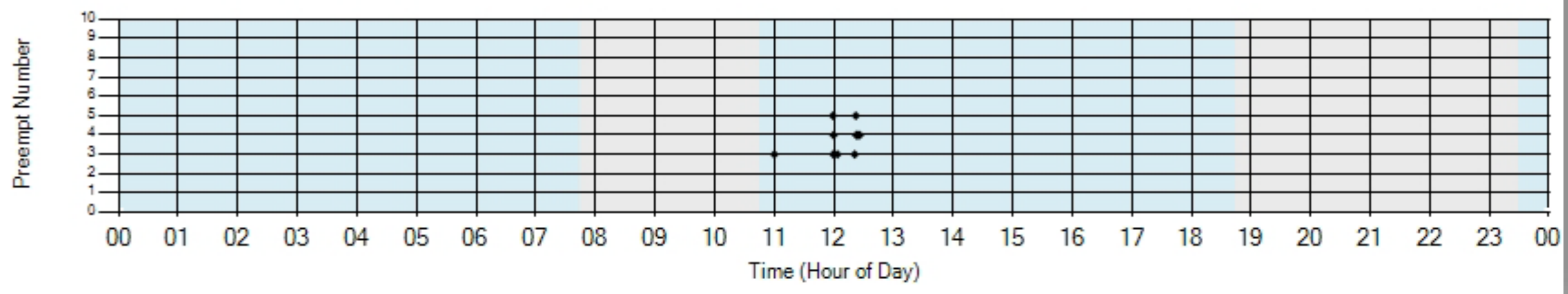




\section{Preemption Details (EMV)}

Preemptor \#3 Event Details

Geneva Pkwy \& 400 S - SIG\#6312

$12 / 9 / 2015$ 0:00 to $12 / 9 / 201523: 59$

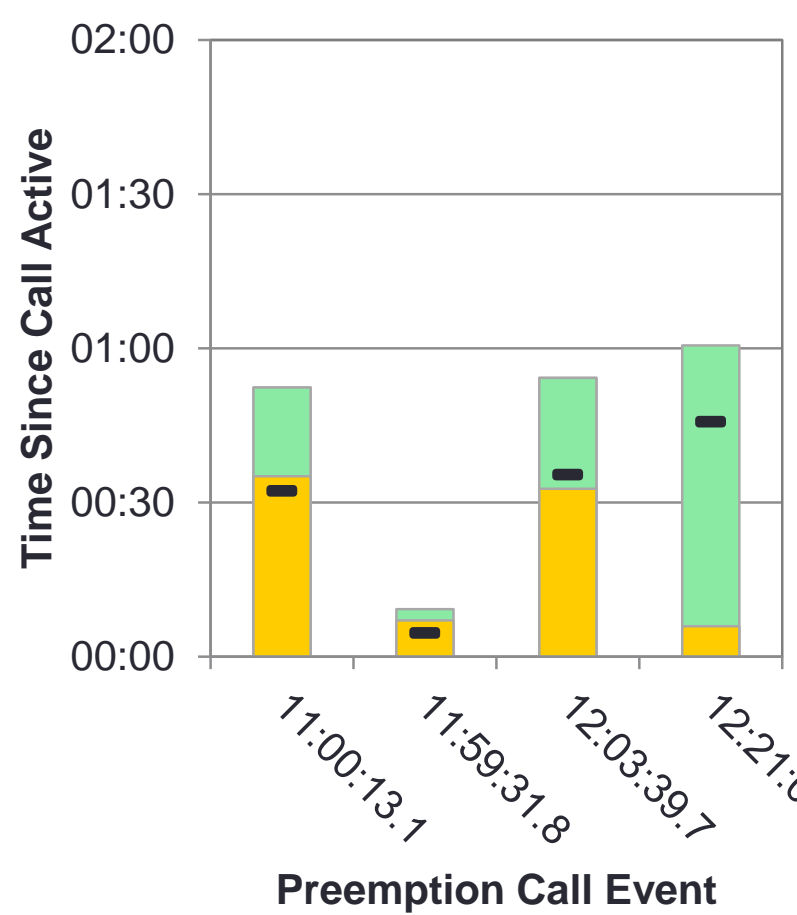

Preemption Call Event
Preemptor \#4 Event Details

Geneva Pkwy \& 400 S - SIG\#6312

12/9/2015 0:00 to 12/9/2015 23:59
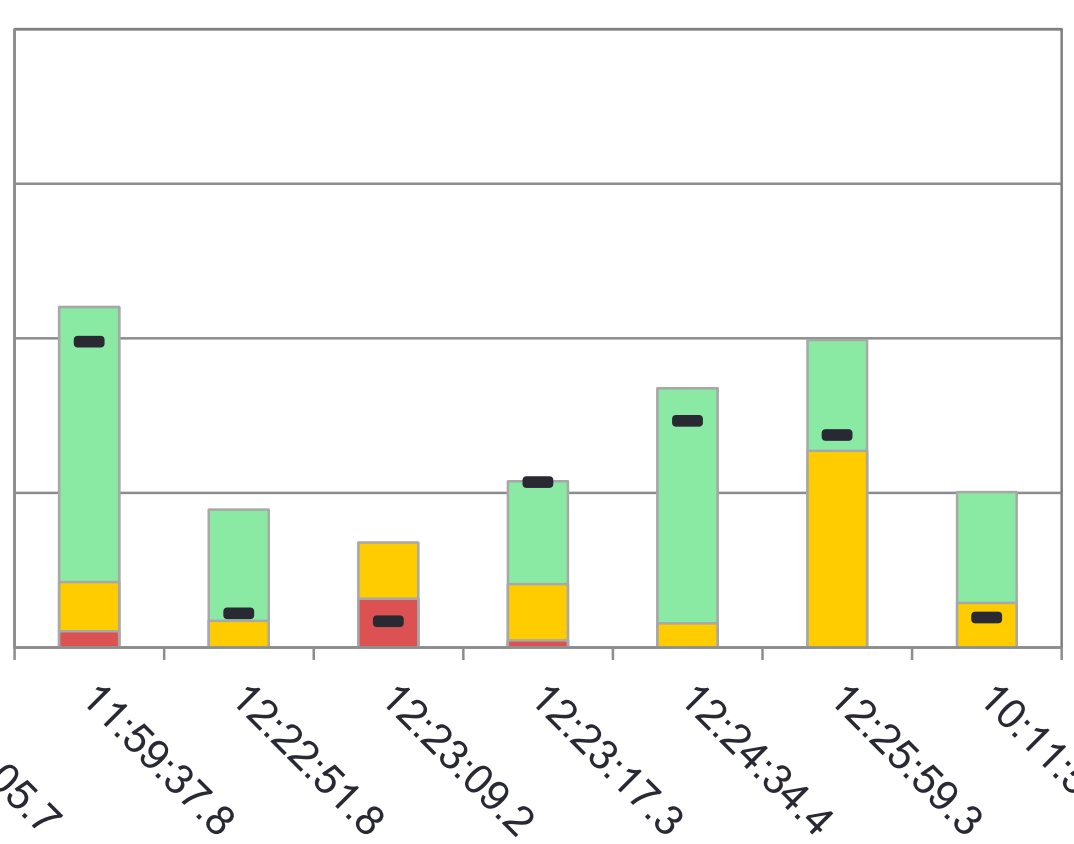

Preemption Call Event
EVENT CODES

102 - Preempt Call On 104 - Preempt Call Off

105 - Preempt Entry Started

107 - Begin Dwell

110 - Preempt Max Out

111 - Begin Exit Interval

Preemptor \#5 Details

Geneva Pkwy \& $400 \mathrm{~S}$

$12 / 9 / 2015$ 0:00 to $23: 59$

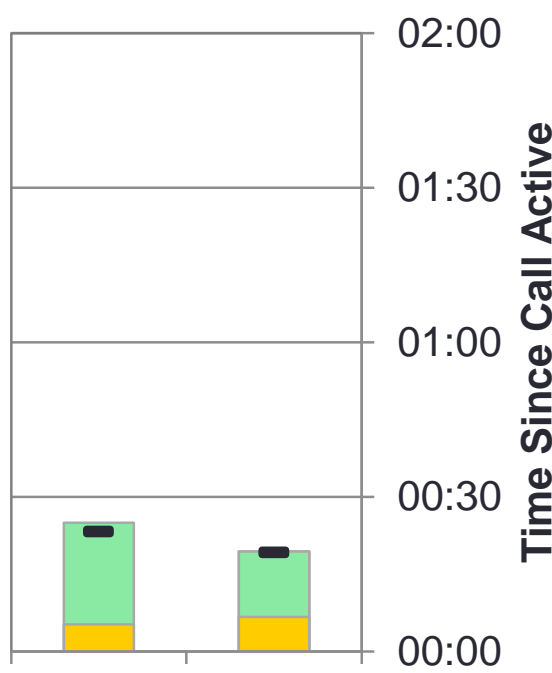

In Step $\quad$ End Dwell $\quad \square$ Time to Service $\quad$ Entry Delay $\quad$ Call End $\times$ Call Max Out $\quad$ Track Clear

$\bullet$ Gate Down 


\section{Transit Signal Priority Requests \& Services}

\section{Priority Request}

3500 S \& 3450 W - SIG\#7284

$12 / 9 / 20150: 00$ to $12 / 9 / 201523: 59$
EVENT CODES

112 - TSP Check In

113 - Early Green

114 - Extend Green

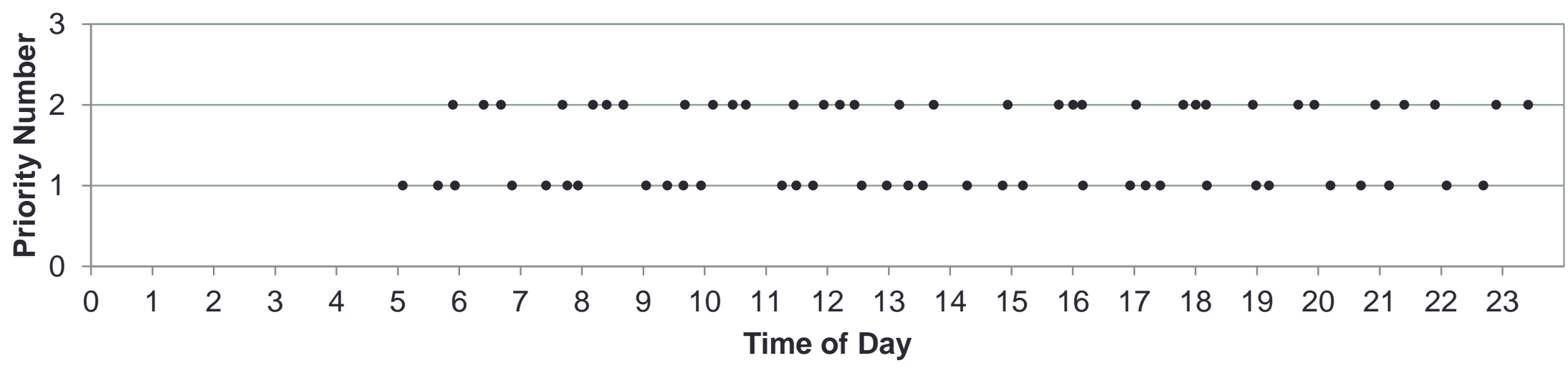

Priority Service

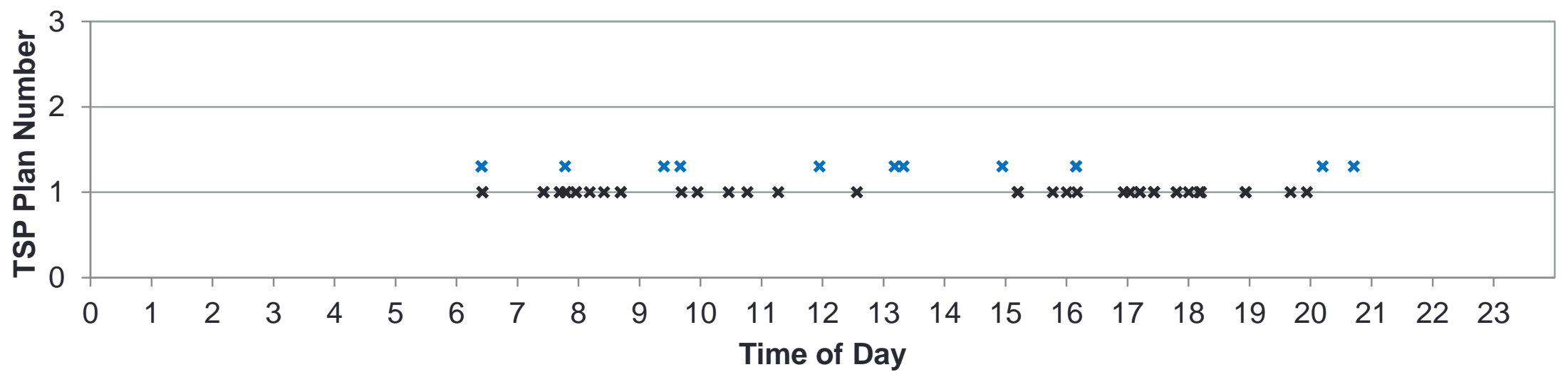

× Early Green × Green Extend 


\section{Transit Signal Priority - Request Duration}

EVENT CODES

112 - TSP Check In

115 - TSP Check Out

Transit Signal Priority Request Duration

3500 S \& 3450 W - SIG\#7284

$12 / 9 / 2015$ 0:00 to $12 / 9 / 201523: 59$

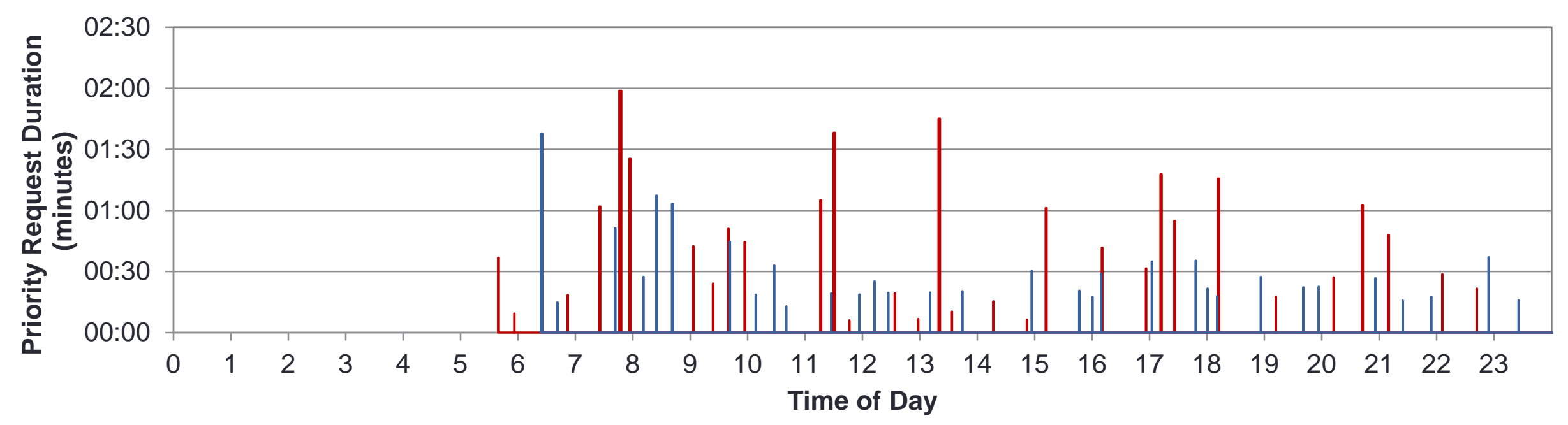

— TSP \#1 — TSP \#2 


\section{Transit Signal Priority - Request Duration}

EVENT CODES

112 - TSP Check In

115 - TSP Check Out

Transit Signal Priority Request Duration

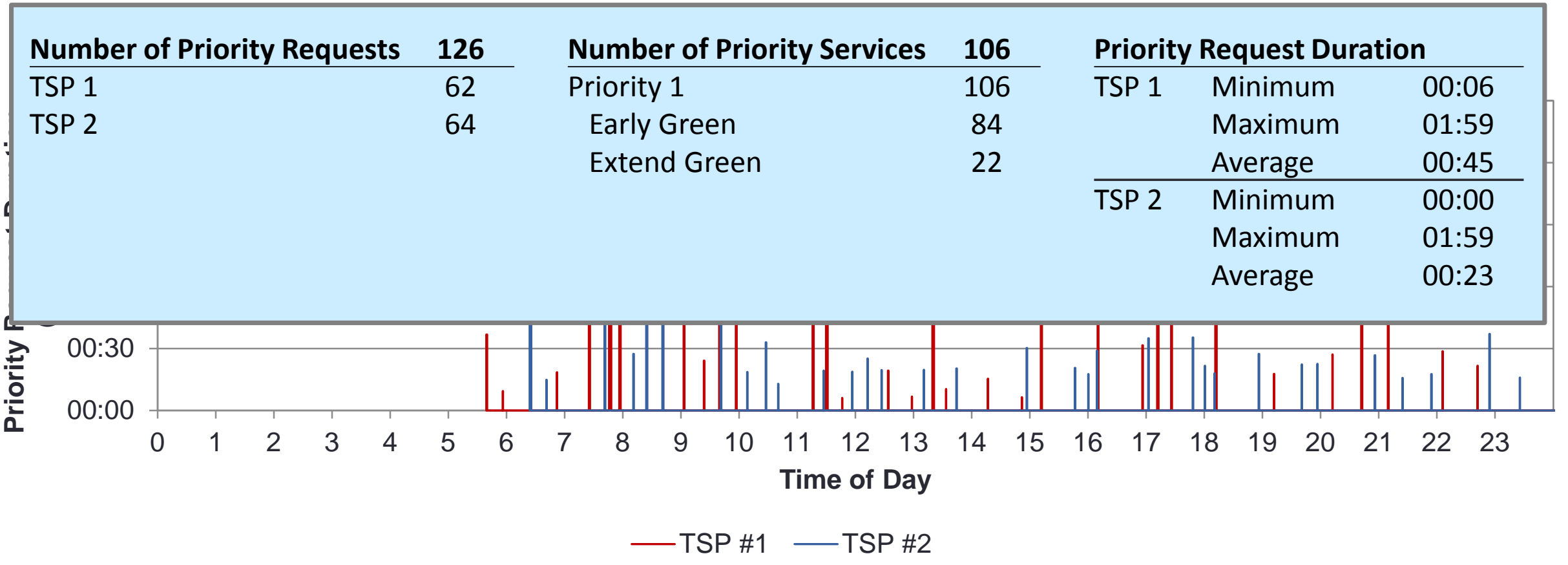




\section{Transit Signal Priority - Request Duration}

EVENT CODES

112 - TSP Check In

115 ton rhack Out

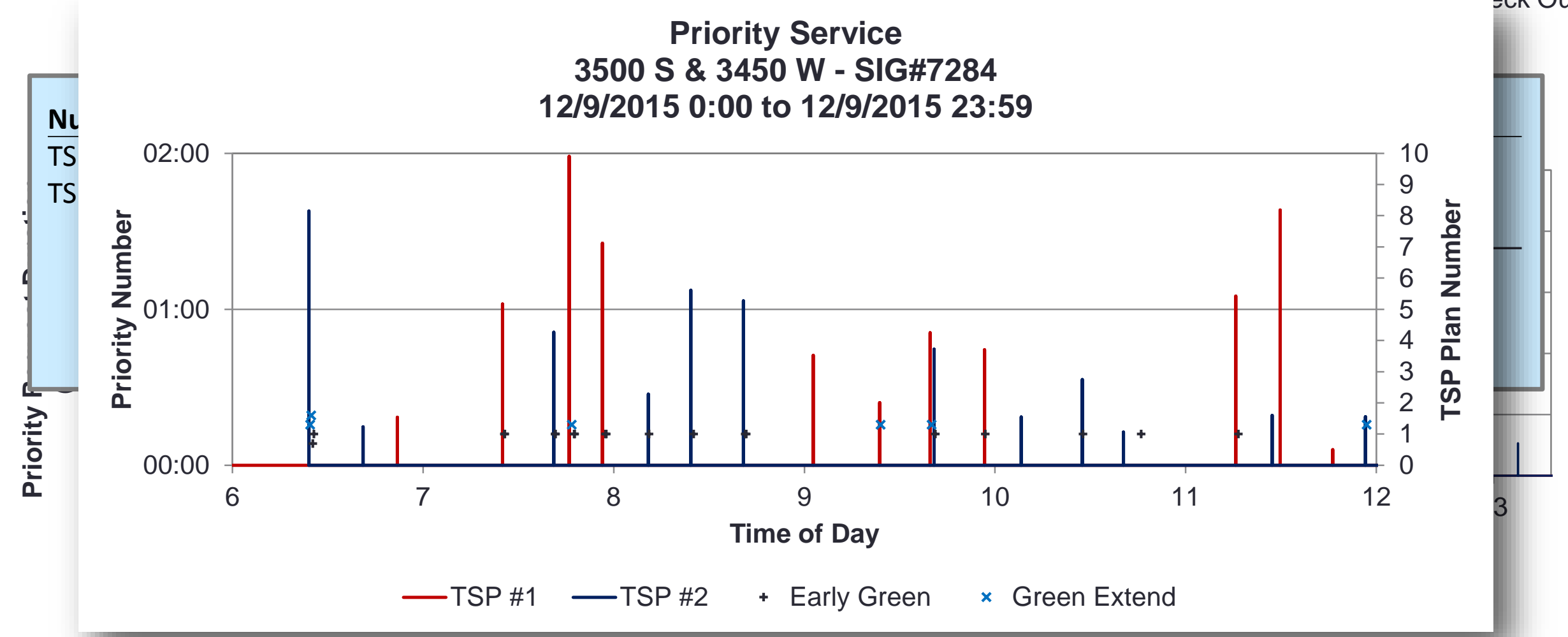




\section{Case Study: Impact of Fully Actuated Coordination on Arrivals on Green}

\author{
US-89 \& $200 \mathrm{~N}$ - SIG\#5382 \\ $11 / 11 / 2015$
}

T $P$ Plan 1 itד 138.2-85 P..T 87.5-85 Percentile Split 127.5-85 Percen...11 ᄀ 126.2 Avg. S... $7^{9.5 \% \text { GapOuts }}$ $0.0 \%$ GapOuts 119.2 Avg. Split 71

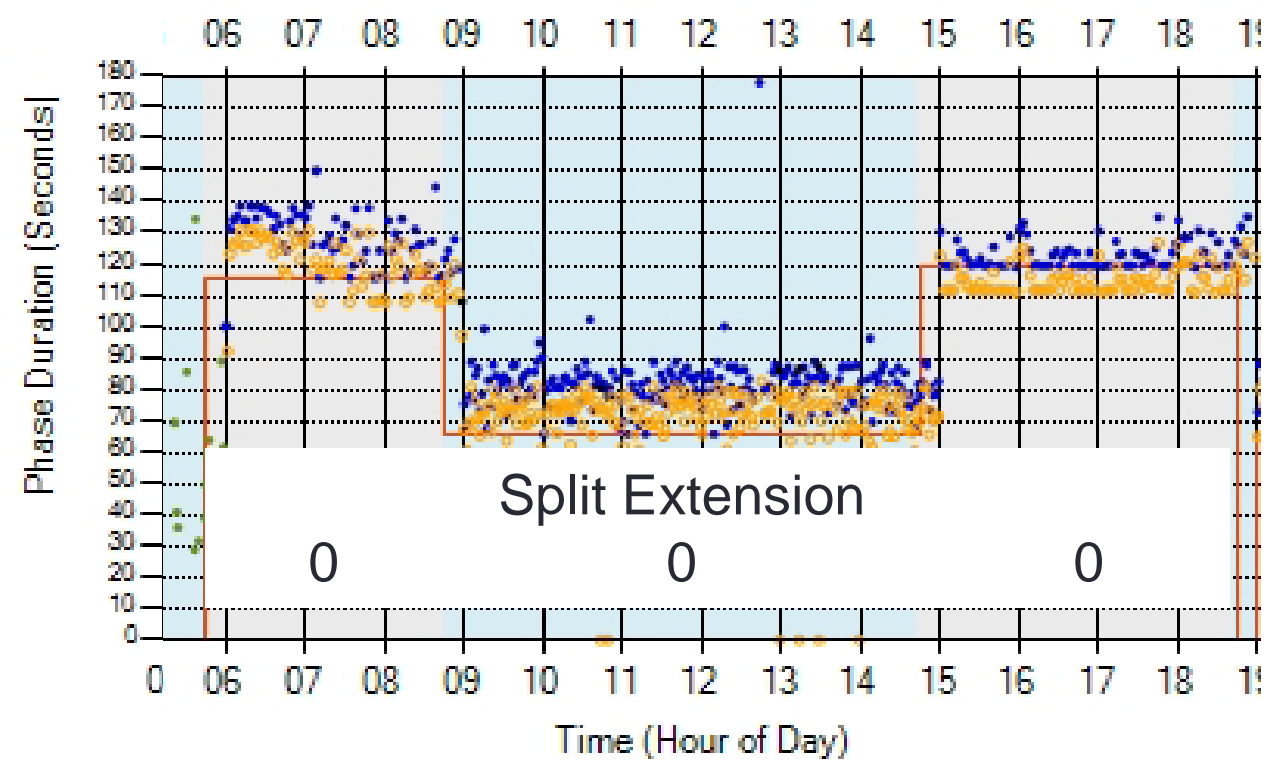

US-89 \& $200 \mathrm{~N}-\mathrm{SIG} \# 5382$ $1 / 13 / 2016$

Plan 7

Plan 13

it 7 T $146.4-85$ Plan

ᄀ 132.0 Avg. S...

$780.3 \%$ GapO

83.7 Avg. Split

$94.8 \%$ GapOuts

133.1 - 85 Percen...T - 121.8 Avg. Split ${ }^{6}$

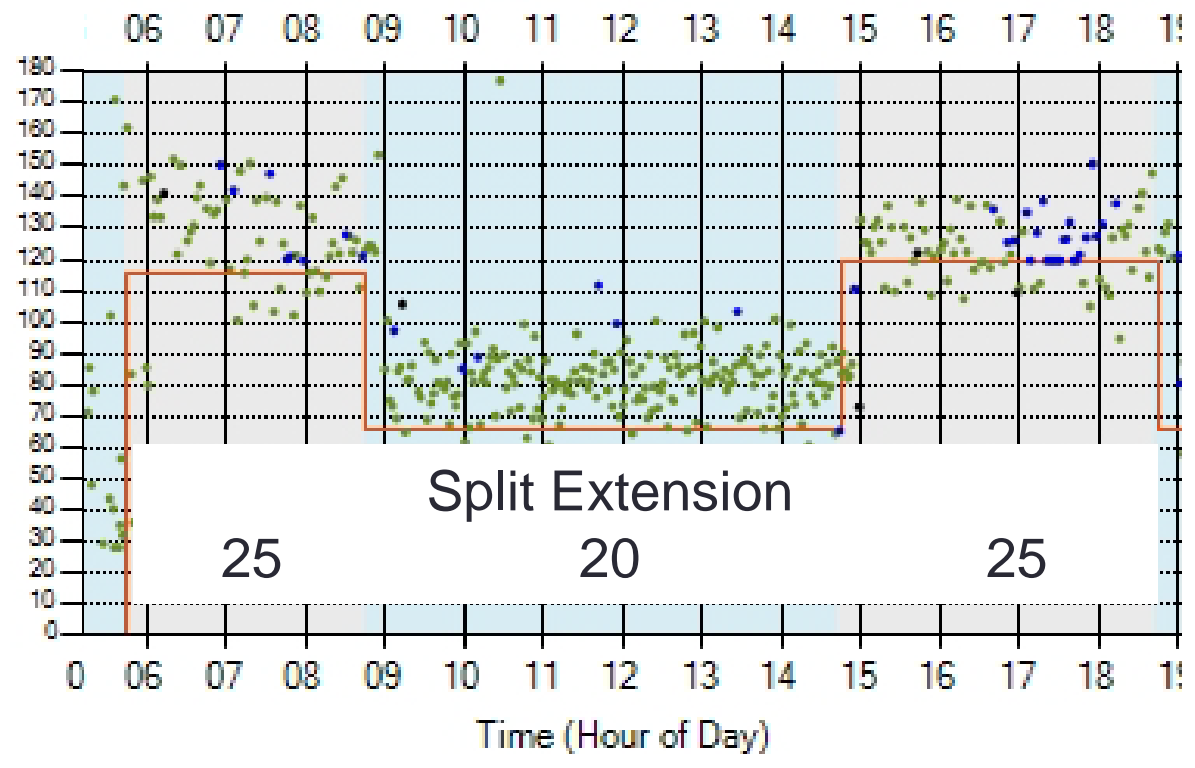


US-89 400 N Layton / 200 N Kaysville Signal 5382 Phase: 2 Northbound Wednesday. November 11, 2015 12:00 AM - Wednesday. November 11, 2015 11:59 PM
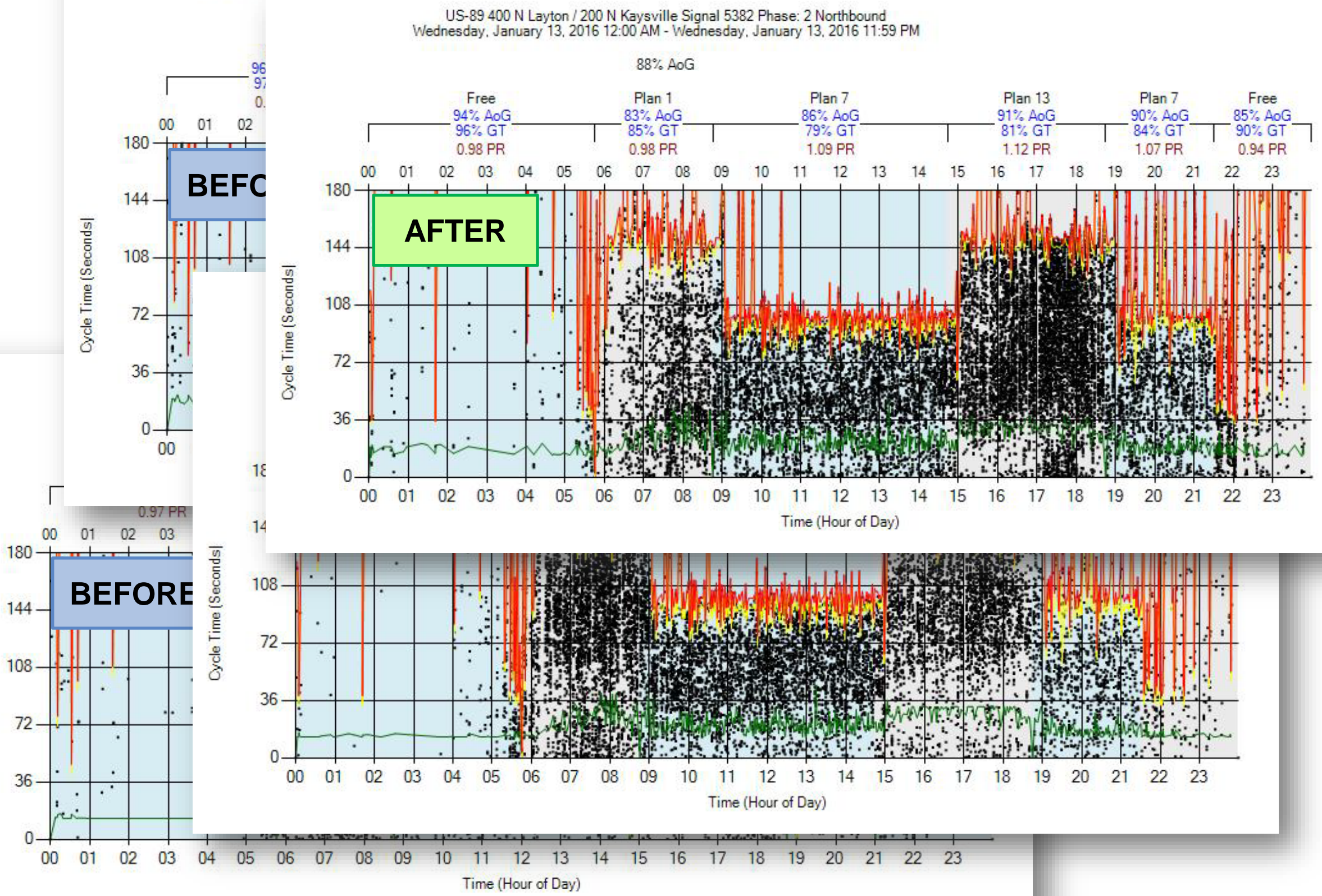
IS-89 $400 \mathrm{~N}$ Layton / $200 \mathrm{~N}$ Kaysyille Signal 5382 Phase: 2 Northbound

Wednesday, November 11, 2015 12:00 AM - Wednesday. November 11, 2015 11:59 PM

US-89 $400 \mathrm{~N}$ Layton $1200 \mathrm{~N}$ Kaysville Signal 5382 Phase: 2 Northbound

Wednesday, January 13, 2016 12:00 AM - Wednesday, January 13, 201611.59 PM
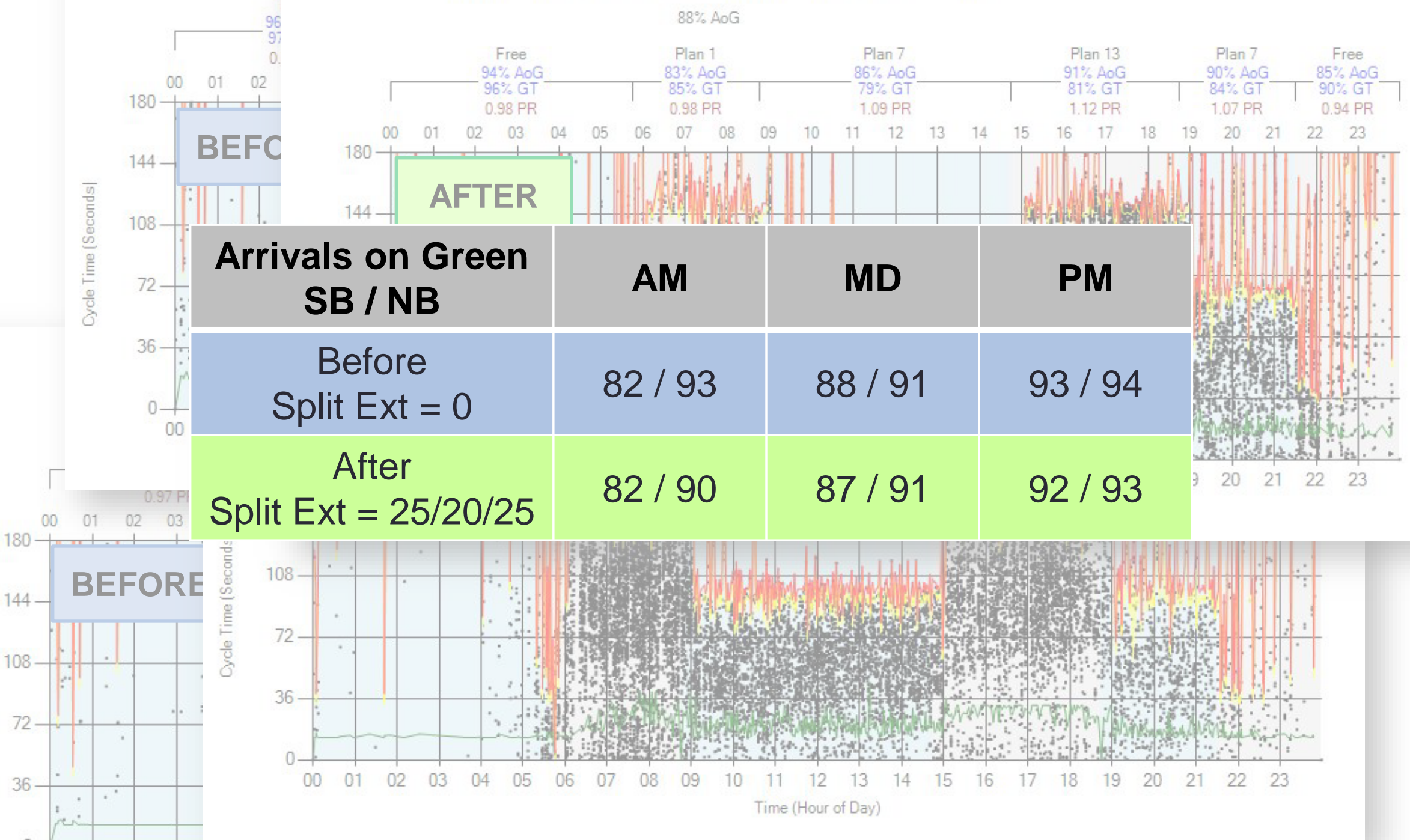

$\begin{array}{llllllllllllllllllllllll}00 & 01 & 02 & 03 & 04 & 05 & 06 & 07 & 08 & 09 & 10 & 11 & 12 & 13 & 14 & 15 & 16 & 17 & 18 & 19 & 20 & 21 & 22 & 23\end{array}$

Time (Hour of Day) 